\title{
El pliego actual y sus implicaciones económicas
}

RAFAel MARIa cuitart, Presidente de la Comisión Interministerial del Pliego del Cemento

Cuando hace algunos meses recibí la invitación de participar en estos sextos Coloquios de Directores y Técnicos de Fábricas de Cemento, que me hizo el Director del Instituto Eduardo Torroja de la Construcción y del Cemento, mi buen amigo Paco Arredondo, no puedo negar la satisfacción que me produjo. Pero a continuación, al sugerirme el tema de "El Pliego actual y sus implicaciones económicas" surgió en mi la preocupación. La segunda parte del título -implicaciones económicas- se presta a un desenfoque fácil si se pretende ligar toda la problemática económica del sector -léase "precios"con el Pliego, que no debemos olvidar que es de prescripciones "técnicas".

Pero yo comprendía que este enfoque era el que V.ds. desearían, por consideraciones pragmáticas y realistas que nadie puede olvidar, conociendo los graves problemas económicos que gravitan sobre el sector.

No obstante, el tiempo que faltaba para el Coloquio me hizo concebir la esperanza de que a estas fechas sus problemas económicos generales estuvieran resueltos y pudieramos reunirnos para comentar las soluciones adoptadas y centrar el tema en un análisis de los efectos económicos directos, con un enfoque académico y técnico.

Pero desgraciadamente para todos y, muy especialmente para el sector de la industria del cemento, su problema de precios sigue sin resolver a pesar del tiempo transcurrido. Esto me obliga a cambiar el enfoque pensado y abordar el tema con el máximo realismo, aun desbordando el condicionante del nuevo Pliego, tratando de buscar entre todos soluciones viables. Por ello voy a reducir al mínimo mi exposición y tratar, por el contrario, de suscitar una especie de tormenta de ideas que pueda provocar entre todos una chispa que ilumine la solución: voy a hablar poco y a escuchar mucho.

\section{Análisis del Pliego de Cementos RC-75}

No creo necesario realizar una descripción del nuevo Pliego de Cementos RC-75, ya que considero indudable el pleno conocimiento del mismo por todos los presentes. Por ello, debemos centrarnos en las ideas básicas que la Comisión redactora consideró que debían presidir su trabajo.

Por supuesto, el anterior Pliego no necesitaba cambios de fondo, ya que su aplicación no había suscitado problemas apreciables y estaba en línea en general con los Pliegos vigentes en otros países con nivel tecnológico comparable al nuestro. Por tanto, el nuevo 
Pliego debía redactarse bajo una idea general de retocar detalles, sin cambios sustanciales. $\mathrm{Y}$ estos retoques deberían encaminarse a lograr varios objetivos:

- realizar un ajuste de definiciones que clarificase los distintos tipos de cementos, suprimiendo algunos que eran prácticamente inexistentes en el mercado;

- en esta misma línea, algún cambio terminológico, como la equívoca denominación de cemento resistente a las aguas selenitosas, cuyo nombre ha dado lugar a alguna curiosa anécdota;

- tipificar la clasificación de cementos en tres órdenes sucesivos: tipo, clase y categoría y establecer las designaciones a partir de las mismas;

- con esto, teóricamente al menos, se reduce el número de cementos diferentes en el mercado, aunque sólo teóricamente, ya que en la práctica dos fabricantes distintos pueden presentar en el mercado dos cementos con igual designación aunque su composición no sea idéntica; con ello, la variedad de cementos puede ser infinita, de tal modo que cada fabricante tiene opción de elegir los que puede producir en forma óptima ;

- supresión de cementos de baja resistencia, como el P-250, que, prácticamente había desaparecido del mercado porque la modernización de nuestras fábricas no permitía producirlo en condiciones económicas;

- paralelamente, la creación de cementos de mayor resistencia con la tipificación de la categoría 550 en varios tipos;

- y sin embargo, ampliar la gama de los cementos con adiciones inertes, el C-200 con composición de hasta sólo el $65 \%$ de clínker para usos no estructurales y de varios tipos con adiciones activas en la categoría 250, concretamente el siderúrgico S-III-250 con contenido de clínker entre 20 y $50 \%$ y los puzolánicos PUZ-I-250 y PUZ-II-250, — con contenido de clínker inferior al 80 \% y sin limitación mínima-, con los cuales quedarán ampliamente cubiertas las necesidades estructurales que requieran sólo resistencias de $250 \mathrm{kp} / \mathrm{cm}^{2}$ a 28 días;

- simplificación de las especificaciones físicas, mecánicas y químicas, ajustando algunas limitaciones y suprimiendo otras que se habían manifestado como inoperantes o innecesarias ;

- tratar de potenciar los cementos con adiciones activas para permitir utilizar recursos existentes - puzolanas, cenizas volantes y escorias-, y reducir con ello el consumo de productos petrolíferos, ante la coyuntura mundial del petróleo.

- y por último, y esto merece ser tratado aparte, la modificación esencial del P-350, al no admitir en su composición las adiciones inertes.

Quiero advertir previamente que cuanto diga respecto al P-350 es prácticamente aplicable, mutatis mutandi, al P-450; por lo cual, en honor a la concisión prescindiré de referirme a este último.

La denominación P-350 del nuevo Pliego, que realmente obedece a una clara razón sistemática del método seguido para establecer las designaciones, va a tener, como veremos luego, inconvenientes prácticos, de carácter no técnico, al utilizar la misma sigla para dos cementos diferentes, según el Pliego de que se trate, máxime cuando el P-350 del antiguo Pliego, que es el de mayor consumo en el mercado, no tiene una correspondencia estrictamente directa con ningún cemento del nuevo Pliego.

El antiguo P-350 puede tener en su composición hasta un $10 \%$ de adiciones, además del regulador de fraguado. Pero no es obligatorio que contenga estas adiciones y éstas pueden ser de hecho inertes o activas. Por tanto, según la composición de cada fábrica, un 
P-350 del antiguo Pliego puede corresponder realmente, según los casos, en el nuevo Pliego con el P-350, con el PA-350, e incluso teóricamente con el C-200, si éste tiene poca adición inerte y da una resistencia muy superior a la requerida.

Sin que esto represente crítica para la Comisión del Pliego, a la que pertenezco y soy plenamente solidario con ella, ya que es más fácil ver las dificultades a posteriori que a priori, creo que hubiera sido preferible conservar la denominación antigua P-350 para el correspondiente cemento del anterior Pliego y haber creado el nuevo cemento con una denominación diferente. Durante la transición habrían podido coexistir ambos sin equívoco, y con el tiempo hubiera ido desapareciendo el viejo P-350 como desapareció sin mayores problemas el P-250.

Quiero aprovechar esta oportunidad para señalar claramente que cuanto he dicho o diga aquí lo hago plenamente a título personal, con mi estricto criterio profesional, sin comprometer en absoluto el criterio de la Comisión del Pliego de Cemento ni de cualquier otro Organismo en el que yo pueda intervenir o pertenecer. Es evidente que si yo tengo un criterio actuaré siempre con arreglo a él, pero el criterio superior de un Organismo prevalecerá lógicamente sobre el mío, si son discrepantes.

\section{Situación y tendencias del mercado de cemento con anterioridad al Pliego RC-75 y posible incidencia de éste}

Si me lo permiten, vamos a dedicar unos minutos a contemplar la situación del mercado de cemento con anterioridad al nuevo Pliego, es decir, hasta 1974, y previsiones que en aquel momento podían deducirse del pasado inmediato.

De la Memoria de la Industria del Cemento, publicada por el Ministerio de Industria, completada con alugnos datos de la correspondiente a 1974, todavía en prensa, y de la de 1975 aún en elaboración, extractamos los siguientes cuadros: I - Producción de clínker normal, y II - Producción de cemento.

\section{CUADRO I}

Producción de clínker normal

\begin{tabular}{ccc}
\hline Año & Producción & $\begin{array}{c}\text { Crecimiento } \\
(\%)\end{array}$ \\
\hline 1968 & 13.371 .398 & - \\
1969 & 14.387 .565 & 7,6 \\
1970 & 14.412 .655 & 1,7 \\
1971 & 14.628 .975 & 1,5 \\
1972 & 17.383 .077 & 18,8 \\
1973 & 19.778 .969 & 13,8 \\
1974 & 21.251 .963 & 7,4 \\
1975 & 22.463 .857 & 5,7 \\
\hline Crecimiento medio acumulativo $\ldots$ & 7,7
\end{tabular}




\author{
CUAD R O II
}

Producción de cemento

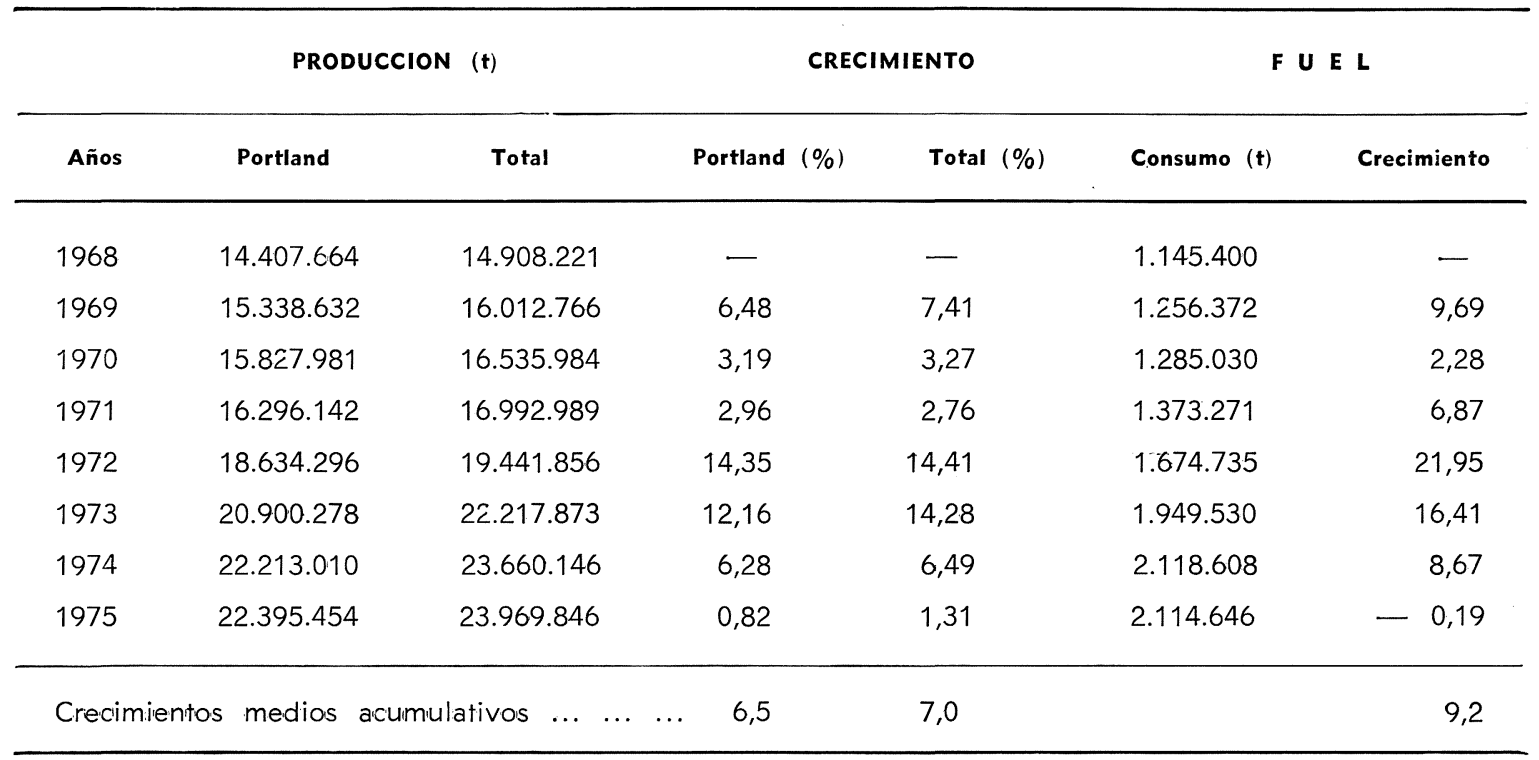

Fuente: Ministerio de Industria - Memoria de la Industria de! Cemento.

En los cuadros vemos las series históricas desde 1968 hasta 1975 de producción de clínker, de cemento portland y total, así como los consumos de fuel. Hemos calculado para las cuatro series históricas los crecimientos anuales y medios acumulativos.

Las series de 1968 a 1975, inclusive, arrojan los siguientes crecimientos medios acumulativos:

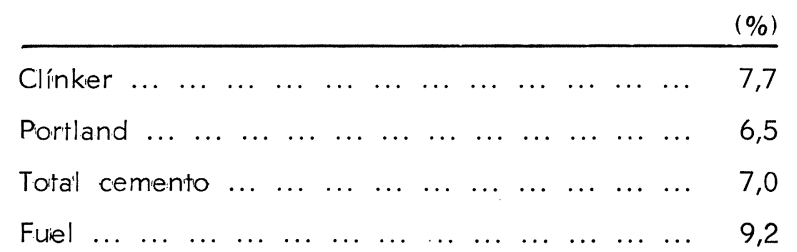

Pero, aparte de ser sobradamente conocido por todos, el simple examen de los citados cuadros muestra que en 1975 se ha roto la tendencia, precisamente en el año en que se aprueba el Pliego, sin ninguna relación de causalidad, pero con adversa incidencia en su efectividad y aplicación, como luego veremos.

Por esto obtenemos estos valores de los crecimientos medios acumulativos, prescindiendo en su cálculo del año 1975, con lo que resulta para las series de 1968 a 1974 los siguientes valores:

\begin{tabular}{|c|c|}
\hline & $(\%)$ \\
\hline $\begin{array}{lllllllllllll}\text { Clínker } & \ldots & \ldots & \ldots & \ldots & \ldots & \ldots & \ldots & \ldots & \ldots & \ldots & \ldots & \ldots\end{array}$ & 8,0 \\
\hline $\begin{array}{llllllllllll}\text { Portland } & \ldots & \ldots & \ldots & \ldots & \ldots & \ldots & \ldots & \ldots & \ldots & \ldots & \ldots\end{array}$ & 7,5 \\
\hline $\begin{array}{lllllllllll}\text { Total cemento } & \ldots & \ldots & \ldots & \ldots & \ldots & \ldots & \ldots & \ldots & \ldots & \ldots\end{array}$ & 8,0 \\
\hline
\end{tabular}

Debe señalarse también el crecimiento, año a año del consumo de fuel superior al crecimiento de la producción, como consecuencia de la transformación tecnológica del sector que va incorporando rápidamente a la producción nuevas fábricas con la más moderna tecnología. No pensamos en absoluto en un crecimiento del consumo de fuel por tone- 
lada producida con este combustible; pero como las nuevas plantas lo utilizan exclusivamente, cada año es menor la proporción de cemento fabricado con carbón respecto a la producción total, con lo cual el consumo de fuel crece casi todos los años varios puntos por encima del crecimiento de la producción de cemento.

Dejemos por un momento esta panorámica histórica y veamos qué hubiera ocurrido, de continuar las tendencias, y en una economia de mercado, al entrar en vigor el nuevo Pliego de Cementos RC-75.

Lógicamente cada fabricante habría estudiado cuáles de los nuevos tipos y clases resultarían más rentables para sus fábricas, dado su emplazamiento en relación con las fuentes de abastecimiento de las adiciones activas admitidas.

Teniendo en cuenta el bajo coste en origen de estas adiciones activas, la distancia de transporte tiene una influencia decisiva en la elección. Una distancia, cuyo coste de transporte unido al coste en origen igualase al coste del propio clínker, haría prohibitiva la utilización. Por debajo de esta distancia la fábrica encontraría ventaja en el caso de la adición activa, ventaja que se traduciría en un mayor radio de distribución de su cemento frente a otros competidores que no pudiesen obtener esta ventaja, o por el contrario, reducción de su radio de distribución frente a competidores que tuviesen la adición activa a menor distancia, o, en resumen, a menor coste.

Por tanto, salvo otras alteraciones en los costes de producción debidos a causas ajenas al Pliego -inflación, convenios colectivos, coste de nuevas inversiones, etc.- éste produciría un efecto inmediato de modificar las áreas de distribución de cada fábrica y una reducción general de los precios de venta.

La modificación de las áreas de distribución no crearían graves problemas a ninguna fábrica ya que en general en todas las áreas geográficas del país existe una posible disposición de una adición activa — puzolana, ceniza volante o escoria-, aunque evidentemente en cada zona habría que elegir la más próxima y prescindir de tipos o clases de cemento que requieran otra adición activa más distante.

El consumo del P-350 disminuiría considerablemente, sustituido por tipos más económicos, quedando reducido su uso a aquellos casos en que técnicamente se requiriese.

$\mathrm{Y}$ otros efectos inducidos y muy favorables se producirían. Con la misma cantidad de clínker podría producirse del orden de un $15 \%$ más de PA-350 que de P-350; para el PUZ-350 y S-I-350, este porcentaje sería del orden del $30 \%$ y muy superior para el S-II-350. En resumen, con mucha prudencia puede estimarse que la capacidad de producción del sector podría aumentar del orden de un $20 \%$ con una moderada inversión, ya que no requeriría ampliar más que la molienda y probablemente silos por la presencia de mayor diversidad del producto.

Si comparamos las importantísimas inversiones realizadas en los últimos años para obtener el crecimiento anual acumulativo del $8 \%$ que hemos contemplado, podemos deducir la enorme ventaja del nuevo Pliego que permitiría atender la continuación de este crecimiento por más de dos años con inversiones muy reducidas.

$\mathrm{Y}$ al mismo tiempo este aumento de producción nos llevaría aparejado el correspondiente incremento del consumo de fuel, con lo cual la incidencia del Pliego en la economía del país, a través de la balanza de pagos, sería considerable, tanto si se optase por reducir las importaciones como si se prefiriese aumentar la producción estabilizando el consumo de fuel. No se olvide que en los últimos años el consumo ha rebasado los 2 millones de t/año de fuel, es decir, que estamos hablando de 400.000 t/año de economía, que re- 
presentan entre el 2 y el $2,5 \%$ del consumo total de fuel del país; su valoración en la balanza de pagos es difícil a través de la tonelada compuesta de petróleo, ya que es uno de los combustibes más favorecidos en la imputación de costes, pero si nos limitamos a su valor en pesetas, la economía superaría los 2.000 millones.

\section{Realidad del mercado de cemento al entrar en vigor el Pliego RC-75}

Dejemos ya el hacer economía-ficción para contemplar la dura realidad.

El efecto del Pliego sobre la economía del sector cemento no varía del esquema que hemos establecido en párrafos anteriores. Pero la situación económica general y la del sector en particular han incidido, si no sobre el Pliego, sí sobre su aplicación real.

Si me permiten un juego de palabras, les diría que, después de hablar de las implicaciones del Pliego de cemento sobre la economía, vamos ahora a ocuparnos de las implicaciones de la coyuntura económica sobre el Pliego.

El Pliego entra en vigor cuando la economía ha quebrado su línea expansiva, y esto hace que la demanda se haya estabilizado, a pesar de la fuerte exportación que el sector ha venido creando en los últimos años y que ahora le ha permitido sostener su producción.

Las premisas de la coyuntura económica que han incidido sobre la aplicación del Pliego RC-75, a las que nos referimos, podrían sintetizarse en las siguientes:

1. Fijación del precio del cemento en fábrica en régimen de precios autorizados.

2. Nivel de precios del cemento.

3. Petición del sector de reconocimiento en su escandallo del mayor coste de amortizaciones y gastos financieros.

4. Retracción del mercado como consecuencia de la crisis económica general.

En el régimen de precios autorizados se fijan éstos por la Administración, a la vista de la petición y datos suministrados por el sector o bien por las empresas individualmente. Dadas las características de uniformidad del producto es lógico que la industria del cemento haya presentado siempre sus peticiones de precios con carácter sectorial.

Por otra parte hay que señalar que los precios autorizados tienen el carácter de máximos, quedando las empresas en libertad de operar por debajo de ellos, circunstancia que, si bien puede darse cuando un precio ha sido recién autorizado, es prácticamente imposible que ocurra al transcurrir cierto tiempo, cuando el efecto inflacionista de los costes ha ido erosionando el margen inicial con el que pudieran contar ciertas empresas mejor situadas en el sector.

La Administración había fijado precios para tres tipos de cementos: P-250, P-350 y blanco. Dada la poca incidencia de éste último y la progresiva desaparición del primero, prácticamente sólo estaba fijado el precio del portland P-350. Los otros tipos de cemento operaban realmente como productos de encargo, cuyo precio se negociaba entre comprador y vendedor, y tengo entendido que como orientación y techo se venía tomando el precio del P-350.

Las últimas modificaciones del precio del cemento habían tenido lugar con la limitación establecida por Decreto de repercutir sólo los aumentos de coste por salarios y materias primas; y aunque éstas se consideraron con amplitud admitiendo como tales 
los combustibles y energía en todas sus formas, dicha restricción no permitió considerar las mayores amortizaciones motivadas por inversiones recientes ni los costes financieros inherentes a las mismas, tal como lógicamente solicitaba el sector.

\section{Actual legislación de precios}

El reciente Real Decreto-Ley 18/76 sobre medidas económicas, publicado el pasado día 11* en el Boletín Oficial del Estado, abre una posibilidad para esta petición del sector. En su artículo $2 .^{\circ}$ admite que los incrementos de precios de bienes y servicios sujetos al régimen de precios autorizados o de vigilancia especial podrán recoger las variaciones en los costos de producción, expresión mucho menos restrictiva que la anterior de salarios y materias primas, y en la cual esperamos que se admita el concepto de amortizaciones. No obstante el mismo Real Decreto-Ley establece algunas otras restricciones que incidirán en este reconocimiento de aumento de costes: por una parte, demora hasta el 30 de noviembre* próximo cualquier autorización de aumento de precios, y por otra, limita la repercusión de elevaciones de costes a las posteriores a primero de enero de 1976.

A pesar de estas limitaciones, tengo la esperanza de que el nuevo Real Decreto-Ley constituya un marco suficientemente realista para permitir encontrar solución a este problema de las amortizaciones, que increíblemente está frenando la aplicación del Pliego.

Las últimas disposiciones económicas del Gobierno han añadido una nueva ventaja para los fabricantes si se aplica el nuevo Pliego RC-75. El Real Decreto 2.344/76 de 8 de octubre, con el que se trata de economizar combustibles, fijando precios diferenciales según los consumos, establece un recargo del $50 \%$ en el precio del fuel a partir de un consumo igual al $95 \%$ del realizado durante los últimos doce meses anteriores al $10^{\circ}$ de octubre corriente. Por tanto, si no se mejoran los rendimientos energéticos, lo cual es prácticamente imposible a plazo corto, los fabricantes tienen que reducir su producción al $95 \%$ de la anterior o soportar un aumento de coste, que, para igual producción, representa el $2,50 \%$ del coste del combustible. Sin embargo, esto que es cierto con carácter general puede ser evitado en el caso del cemento mejorando el rendimiento energético - como venimos diciendo- mediante la utilización de adiciones activas que reduzcan la componente de clínker por tonelada de cemento, aumentando incluso la producción total de éste sin incidir en el recargo del precio del fuel.

Con el nuevo Pliego RC-75 se presenta la posibilidad de un gran abanico de cementos y, además, la conveniencia de que su elección y adquisición sea fácil, es decir, que existan en el mercado sin necesidad de encargo negociado.

Y para que estos tipos, clases y categorías de cementos existan en el mercado es imprescindible que tengan interés en ello todos los agentes implicados: promotores de la construcción (Administración Pública o propietario privado), técnicos, constructores y, fundamentalmente, fabricantes de cemento. $\mathrm{Y}$ este interés en general vendrá motivado por un beneficio económico.

Por tanto, el beneficio resultante de los nuevos tipos de cemento deberá distribuirse equitativamente entre todos estos escalones de la producción presentes en la actividad de la construcción.

Veamos como dentro de estas disposiciones limitativas del libre mercado debería actuarse para fijar los precios de los nuevos tipos de cementos.

Para fijar el precio de nuevos productos la normativa vigente (D/1531/74 de 22 de mayo que desarrolla el D. L./12/73 de 30 de noviembre) exige la justificación de costes de personal, materias primas $\mathrm{y}$, en general, de los componentes que incidan en la formación del precio, señalando el proceso de producción y referencia a otros bienes similares existentes en el mercado.

Por consiguiente los precios de los nuevos tipos de cemento deben obtenerse relacionándolos con el P-350 del antiguo Pliego, fijado por el Gobierno en régimen de precios auto-

Conferencia pronunciada en octubre de 1976. 
rizados. Y dado el desfase de este precio con la actual situación de costes, es imprescindible la actualización previa o simultánea del mismo.

A partir del precio del P-350 antiguo, y considerando las distintas composiciones de los nuevos tipos, clases y categorías, teniendo en cuenta la proporción de adición activa, su coste en origen, su distancia de transporte y el factor de molienda, es fácil obtener el precio de cada uno de los nuevos cementos.

Pero como venimos diciendo, estos precios diferirán de forma importante para cada fábrica, por lo cual, en nuestro criterio, sería más operativo y equitativo fijar estos precios en régimen de vigilancia especial, a partir del nuevo P-350 o de su clínker en régimen de precios autorizados. Con ello cada fábrica aplicaría el precio más ajustado a su propia realidad de costes.

Si no se acordase el cambio de régimen de "autorizado" a "vigilancia especial" cabría también una solución, que podría consistir en fijar para cada tipo y clase una distancia de transporte de la adición activa y una proporción de ésta razonables y orientativas. Con estos datos se podría fijar un precio autorizado, para cada tipo y clase, aunque esta uniformidad tendría - a mi juicio- el inconveniente de reducir la competencia y no obtener la máxima economía posible derivada del uso de adiciones activas: el fabricante más próximo al suministro de la adición obtendría un beneficio marginal derivado del menor coste del transporte, mientras que en cambio otros, más distantes del suministro de la adición, no podrían competir.

Con los datos que he podido obtener he hecho algunos cálculos y creo poder afirmar que los costes de la mayor parte de los PA-350, PUZ-350 y S-I-350 están entre el 80 y el $90 \%$ del P-350 del nuevo Pliego; y el S-II-350, incluso más bajo. Si consideramos el encareci-

miento que el nuevo P-350 pueda tener respecto al antiguo de igual denominación, los nuevos cementos con adiciones activas pueden tener precios del mismo orden que el actual del P-350; algunos por debajo y algunos por encima, ya que -como he dicho- las variaciones entre fábricas pueden ser importantes.

$\mathrm{Y}$ en estos cálculos he considerado por supuesto una posible revisión de las amortizaciones, aunque no puedo aventurar de momento cuál podría ser su cuantía exacta.

Por tanto, entiendo que el nuevo Pliego, con una aplicación racional de los nuevos tipos, puede permitir absorber, total o al menos en parte importante, las aspiraciones del sector de mejorar las amortizaciones de sus instalaciones.

\section{Posibles medidas para instrumentar una solución}

Como les decía al principio quisiera escuchar muchas ideas para instrumentar una solución a este problema que interesa a todos y que me atrevería a calificar de alto interés económico nacional: la plena aplicación del Pliego RC-75.

Pero para que surjan ideas suele ser útil emplear un detonante: esto quiere ser, nada más solución que propongo. Siguiendo las reglas de la tormenta de ideas no es eficaz que traten de atacar y yo de defender esta solución. Es una de las posibles y sólo pretendo sugerir la iniciativa de cada uno. Traten de mejorarla, lancen ideas, y en una próxima oportunidad, Administración y Sector, deberán analizar, discutir y elegir la óptima. Bien entendido que óptima no debe ser para cada uno la que más se aproxime a nuestras propias ideas, sino la que resuelva mejor las distintas facetas del problema y reúna características de viabilidad. Una solución, teóricamente perfecta, que no sea viable no es una solución eficaz. 
Quiero recordar, antes de resumir, que el Pliego RC-75 es obligatorio en todo el territorio nacional a partir del pasado 1 de junio. $Y$ que en esta situación de inaplicación de lo dispuesto, de "vacatio legis" de hecho, que no de derecho, no podemos continuar. Cualquier accidente relacionado con el empleo de un cemento, aunque dependa de otras causas, podría tener complicaciones absolutamente innecesarias, pero siempre lamentables, que no ofrecerían ventaja para nadie.

Creo, por tanto, imprescindible que se dicte una disposición complementaria al Decreto de Presidencia del Gobierno 1964/75 de 23 de mayo, por el que se aprueba el "Pliego de Prescripciones Técnicas Generales para la Recepción de Cementos RC-75", que prorrogue la vigencia del Pliego del 64 por un año, durante el cual estarían en vigor simultáneamente tanto el Pliego RC-75 como el del 64, pudiendo ser optativamente utilizado cualquiera de ellos.

Con esta medida quedaría legalizada la situación de proyectistas, constructores y fabricantes durante dicho período, en el que deberán tomarse las medidas necesarias para conseguir el pleno abastecimiento del mercado con los nuevos tipos de cemento.

$\mathrm{Y}$ como hemos dicho, para que estos nuevos tipos de cemento salgan al mercado es necesario fijarles precio, para lo cual, resumiendo lo expuesto anteriormente, debe actualizarse por los Organos competentes, al amparo de las nuevas disposiciones sobre precios, el del antiguo P-350 dentro del régimen de precios autorizados, así como de su clínker, y del nuevo P-350.

Declarar con precios de "vigilancia especial" a los cementos con adiciones activas y compuestos, fijado para ellos en este régimen un límite superior igual al del P-350 antiguo actualizado, por encima del cual no deberían ser fabricados por antieconómicos.

\section{Colofón}

$\mathrm{Y}$ con esto termino y quiero pasarles la palabra.

El RC-75 no es, indudablemente, la panacea para todos los problemas del sector, pero utilizado razonable y correctamente creo que puede contribuir a resolver algunos de ellos y aliviar otros, sin deteriorar la ya tan averiada economía nacional e, incluso, colaborando a mejorar situaciones como la de la balanza de pagos, tan afectada por las importaciones de petróleo. 